\title{
ON THE CENTRE AND RESIDUAL FINITENESS OF THE AUTOMORPHISM GROUP OF A GROUP RING
}

\author{
by D. A. R. WALLACE
}

(Received 2nd July 1985)

\section{Introduction}

Let $G$ be a group and let $\operatorname{Aut}(G)$ be its automorphism group. It is notorious that the properties of $\operatorname{Aut}(G)$ do not relate well to the properties of $G$, perhaps the only two general results being that if $G$ has a trivial centre then the same is true of $\operatorname{Aut}(G)[2, \mathrm{p}$. 89] and Baumslag's theorem that if $G$ is finitely generated and residually finite then $\operatorname{Aut}(G)$ is also residually finite [1, Theorem 1, p. 117]. In the paper we shall attempt to find analogues of these results for the relationship between the properties of $R(G)$, the group ring of $G$ over a ring $R$, and the properties of Aut $R(G)$, the automorphism of $R(G)$. We prove that if $R(G)$ has a trivial centre then Aut $R(G)$ has a trivial centre. We establish the analogue, Theorem 2.3, of Baumslag's theorem by ring-theoretic methods; our original proof used properties of group rings, the present simplified proof we owe to the referee. As an example we calculate $\operatorname{Aut} \mathbb{Z}(G)$ in the case that $G$ is the direct product of two cyclic groups, one of infinite order and the other of order 5. This calculation will, it is hoped, give some indication of the difficulties in determining automorphisms of the group ring of an infinite group.

In our notation it will be assumed that $R, S$ are rings with identities. Aut $R(G)$ is the group of $R$-linear automorphisms of $G$ and $A u t_{+} R(G)$ is the subgroup of Aut $R(G)$ consisting of the augmentation-preserving automorphisms (i.e. if $\varepsilon: R(G) \rightarrow R$ is the augmentation then for all $\theta \in \mathrm{Aut}_{+} R(G)$ and for all $\left.g \in G, \varepsilon(\theta(g))=1\right)$.

\section{Residual finiteness of Aut $R(G)$}

For convenience we state the following definitions for the ring $S$.

Definition. $S$ is said to be finitely generated if there exists a finite non-empty subset $X$ of $S$ such that $S$ is spanned as a $\mathbb{Z}$-module by the subset consisting of the products of the elements of $X$.

Definition. $S$ is said to be residually finite if for all $x \in S, x \neq 0$, there exists an ideal $I$ of $S$ such that $x \notin I$ and $S / I$ is a finite ring.

For example $\mathbb{Z}$ is both finitely generated and residually finite.

Lemma 2.1. Let $S$ be finitely generated. Let $n \in \mathbb{N}$. Then there exist only finitely many ideals I such that $|S / I| \leqq n$.

Proof. For any $n \in \mathbb{N}$ there exists at most a finite number of non-isomorphic rings 
each consisting of precisely $n$ elements. Let $T$ be one such ring of $n$ elements. Since $S$ is finitely generated there exists at most a finite number of homomorphisms of $S$ into $T$ and so $T$ determines at most a finite number of ideals $I_{1}, I_{2}, \ldots, I_{r}$ (say) such that $S / I_{i}$ is isomorphic to a subring of $T(i=1,2, \ldots, r)$. Let $T^{\prime}$ be an isomorphic copy of $T$. Then the ideals of $S$ determined by $T^{\prime}$, determined in the same manner as those determined by $T$, are again precisely $I_{1}, I_{2}, \ldots, I_{r}$. Thus each finite ring and its isomorphic copies determine in this way at most a finite number of ideals of $S$. Hence, in conclusion, there can exist at most a finite number of ideals $I$ for which $|S / I| \leqq n$.

The proof of the next result parallels the proof of Baumslag's original theorem [1, Theorem 1, p. 117].

Lemma 2.2. Let $S$ be a finitely generated, residually finite ring. Then Aut $S$, the group of automorphisms of $S$, is residually finite.

Proof. Let $\phi \in$ Aut $S, \phi \neq l$. Then there exists $x \in S$ such that

$$
y=\phi(x)-x \neq 0
$$

By hypothesis there exists an ideal $I$ of $S$ such that $y \notin I$ and $S / I$ is a finite ring. Suppose $|S / I|=n$. Then there exists at most a finite number of ideals $J$ such that $S / J \leqq n$. Let $I_{0}$ be the intersection of these ideals $J$. Then $y \notin I_{0}, S / I_{0}$ is finite and $I_{0}$ is a characteristic ideal of $S$. We define a map Aut $S \rightarrow \operatorname{Aut}\left(S / I_{0}\right)$ as follows. For $\theta \in$ Aut $S$ let $\tilde{\theta}$ be defined by

$$
\ddot{\theta}: s+I_{0} \rightarrow \theta(s)+I_{0} \quad(\forall s \in S)
$$

Since $I_{0}$ is a characteristic in $S \tilde{\theta} \in \operatorname{Aut}\left(S / I_{0}\right)$ and the map $\theta \rightarrow \tilde{\theta}(\forall \theta \in$ Aut $S)$ is a homomorphism. Let $W$ be the kernel of this homomorphism. Then (Aut $S$ )/W is isomorphic to a subgroup of the finite group $\operatorname{Aut}\left(S / I_{0}\right)$. Further as $y \notin I_{0}, \delta \neq i$. Thus $\phi \notin W$ and hence the result is proved.

Theorem 2.3. Let $G$ be a finitely generated, residually finite group. Let $R$ be a finitely generated residually finite ring. Then Aut $R(G)$ is residually finite.

Proof. Let $S=R(G)$. Then certainly $S$ is finitely generated. It suffices to prove that $S$ is residually finite for then the result will follow from Lemma 2.2. Let $x \in R(G), x \neq 0$. Suppose

$$
x=\lambda_{1} g_{1}+\lambda_{2} g_{2}+\cdots+\lambda_{r} g_{r}
$$

where $g_{1}, g_{2}, \ldots, g_{r}$ are distinct elements of $G$ and $\lambda_{i} \in R, \lambda_{i} \neq 0(i=1,2, \ldots, r)$. By assumption on $G$ there exists a normal subgroup $H$ of finite index in $G$ such that

$$
\left\{g_{i} g_{j}^{-1}: i \neq j ; i, j,=1,2, \ldots, r\right\} \cap H=\emptyset \text {. }
$$


By assumption on $R$ there exists an ideal $I$ of $R$ for which $R / I$ is finite and

$$
\left\{\lambda_{1}, \lambda_{2}, \ldots, \lambda_{r}\right\} \cap I=\emptyset
$$

The composite homomorphism

$$
R(G) \rightarrow R(G / H) \rightarrow(R / I)(G / H)
$$

maps $R(G)$ into a finite ring and, by the construction of $H$ and $I, x$ is not in the kernel of this composite homomorphism. The result is now established.

Corollary 2.4. Let $G$ be a finitely generated, residually finite group. Then $A u t \mathbb{Z}(G)$ is residually finite.

\section{An example}

Throughout this section we study the example to which allusion was made above.

Example 3.1. (We are indebted to Don Passman for drawing the group $G$ below to our attention.) Let $G=\langle x\rangle \times\langle y\rangle$ be the direct product of the subgroups $\langle x\rangle$ and $\langle y\rangle$ where $x$ has infinite order and $y^{3}=1$. We shall calculate $\operatorname{Aut}(G)$ and $\operatorname{Aut} \mathbb{Z}(G)$ and we begin by calculating the former group.

Let $\theta \in \operatorname{Aut}(G)$. Since $[\theta(y)]^{5}=1$ we have

$$
\theta(y) \in\left\{y, y^{2}, y^{3}, y^{4}\right\}
$$

Since $\theta(x)$ has infinite order and $\theta$ has, of course, to be surjective we have

$$
\theta(x) \in\left\{x, x y, x y^{2}, x y^{3}, x y^{4}, x^{-1}, x^{-1} y, x^{-1} y^{2}, x^{-1} y^{3}, x^{-1} y^{4}\right\} .
$$

It follows that $|\operatorname{Aut}(G)| \leqq 40$. We shall prove that $|\operatorname{Aut}(G)|=40$ by constructing a system of generators and relations for $\operatorname{Aut}(G)$. We shall define our automorphisms as mappings on the generators $x, y$ of $G$ which will extend appropriately and unambiguously to automorphisms of $G$.

We define $\alpha, \sigma, \tau$ by

$$
\begin{array}{lll}
\alpha(x)=x, & \sigma(x)=x y, & \tau(x)=x^{-1} \\
\alpha(y)=y^{2}, & \sigma(y)=y, & \tau(y)=y
\end{array}
$$

and note that $\alpha, \sigma, \tau$ do extend to automorphisms of $G$.

Evidently we have

$$
\alpha^{4}=\sigma^{5}=\tau^{2}=1, \quad \alpha \tau=\tau \alpha, \quad \alpha^{-1} \sigma \alpha=\sigma^{3}, \quad \tau^{-1} \sigma \tau=\sigma^{4}
$$


Thus $\operatorname{Aut}(G)=\langle\alpha, \sigma, \tau\rangle$ is a soluble group of order 40 with $\langle\sigma\rangle$ as a normal 5-Sylow subgroup and with $\langle\alpha, \tau\rangle$ as an abelian 2-Sylow subgroup.

We now consider $\mathbb{Z}(G)$ and calculate $\operatorname{Aut} \mathbb{Z}(G)$. There is an obvious inclusion, namely,

$$
\operatorname{Aut}(G) \subseteq \text { Aut }_{+} \mathbb{Z}(G) \subseteq \text { Aut } \mathbb{Z}(G)
$$

Let $\lambda \in$ Aut $\mathbb{Z}(G)$ be defined by $\lambda(x)=-x, \lambda(y)=y$. We claim that $\mid$ Aut $\mathbb{Z}(G)$ :Aut $+\mathbb{Z}(G) \mid=2$ and that in fact

$$
\text { Aut } \mathbb{Z}(G)=\text { Aut }_{+} \mathbb{Z}(G) \cup \text { Aut }+\mathbb{Z}(G) \lambda
$$

To see this let $\theta \in A u t \mathbb{Z}(G)$. Now for any unit $t$ of $\mathbb{Z}(G)$ we have $\varepsilon(t)= \pm 1$ and so, in particular, $\varepsilon(\theta(x))= \pm 1$ and, as $[\theta(y)]^{5}=1, \varepsilon(\theta(y))=1$. From this it follows that either $\theta \in$ Aut $_{+} \mathbb{Z}(G)$ or $\theta \lambda \in$ Aut $_{+} \mathbb{Z}(G)$ and so we obtain the above coset decomposition. In fact, as we shall establish below,

$$
\text { Aut } \mathbb{Z}(G)=\text { Aut }_{+} \mathbb{Z}(G) \times\langle\lambda\rangle \text {. }
$$

For the present we shall calculate Aut $+\mathbb{Z}(G)$.

As is well known $\mathbb{Z}(\langle x\rangle)$ has only the trivial units $\pm x^{n}(n \in \mathbb{Z})$ but $\mathbb{Z}(\langle y\rangle)$ has nontrivial units, e.g. $1-y^{2}+y^{4}$ is a unit of infinite order for which $\varepsilon\left(1-y^{2}+y^{4}\right)=1-1+1=1$. By a theorem of Sehgal [4, Theorem 3.1, p. 54] the group units of $\mathbb{Z}(\langle y\rangle)$ is isomorphic to

$$
( \pm\langle y\rangle) \times F
$$

where $F$, in this case, is a free abelian group of rank 1 . Let $u$ be a generator of $F$ for which $\varepsilon(u)=1$. Let $\theta \in$ Aut $+\mathbb{Z}(G)$. Since $[\theta(y)]^{5}=1, \theta(y)$ is a unit of order 5 and so

$$
\theta(y) \in\left\{y, y^{2}, y^{3}, y^{4}\right\} \text {. }
$$

Since $\theta(x)$ has infinite order and $\theta$ is surjective we have

$$
\theta(x) \in\left\{x w, x^{-1} w: w \text { is a unit of } \mathbb{Z}(\langle y\rangle)\right\} .
$$

Thus we obtain $\alpha, \sigma, \tau$ as before but we have another generator $\rho$ of Aut $+\mathbb{Z}(G)$ given by

$$
\rho(x)=x u, \quad \rho(y)=y .
$$

We claim that we may suppose that $u$ has been chosen so that $\alpha(u)=y u^{-1}$, a relation which is convenient for subsequent calculations. Clearly $\alpha$ acts as an augmentationpreserving automorphism on the group of units $\mathbb{Z}(\langle y\rangle)$. Thus $\alpha(u)=y^{\delta} u^{ \pm 1}$ for some $\delta \in\{0,1,2,3,4\}$. For the sake of argument suppose that $\alpha(u)=y^{\delta} u$. From our remarks above

$$
1-y^{2}+y^{4} \in\langle y\rangle \times F
$$


and so there exist $p, q$ such that

$$
1-y^{2}+y^{4}=y^{p} u^{q}
$$

Then

$$
\begin{aligned}
1-y^{4}+y^{3} & =\alpha\left(1-y^{2}+y^{4}\right)=\alpha\left(y^{p} u^{q}\right) \\
& =[\alpha(y)]^{p}[\alpha(u)]^{q}=\left[y^{2}\right)^{p}\left[y^{\delta}\right]^{q} \\
& =y^{2 p+\delta q} u^{q}=y^{p+\delta q}\left(y^{p} u^{q}\right) \\
& =y^{p+\delta q}\left(1-y^{2}+y^{4}\right)
\end{aligned}
$$

which is impossible and consequently $\alpha(u)=y^{\delta} u^{-1}$. Let

$$
v=y^{2(1-\delta)} u \text {. }
$$

Then

$$
\langle y\rangle \times\langle u\rangle=\langle y\rangle \times\langle v\rangle
$$

and

$$
\begin{aligned}
\alpha(v) & =[\alpha(y)]^{2(1-\delta)} \alpha(u) \\
& =\left[y^{2}\right]^{2(1-\delta)} y^{\delta} u^{-1} \\
& =y^{4-3 \delta} u^{-1} \\
& =y v^{-1}
\end{aligned}
$$

Hence we may suppose that originally we have chosen an infinite generator $u$ of $\pm\langle y\rangle \times F$ such that

$$
\alpha(u)=y u^{-1}
$$

We now claim that Aut $+\mathbb{Z}(G)=\langle\alpha, \sigma, \tau, \rho\rangle$. First of all from $\left({ }^{*}\right),\left({ }^{* *}\right),\left({ }^{* * *}\right)$ we deduce that

$$
\rho^{-1}(x)=x u^{-1}, \quad \alpha^{2}(u)=y u, \quad \alpha^{3}(u)=y^{3} u^{-1} .
$$

To prove that Aut $+\mathbb{Z}(G)=\langle\alpha, \sigma, \tau, \rho\rangle$ we have to show that if $\pi \in A_{u t} \mathbb{Z}(G)$ then $\pi \in\langle\alpha, \sigma, \tau, \rho\rangle$. From remarks above we have $\pi(x)=x^{ \pm 1} z, \pi(y)=y^{\mu}$ where $z$ is some unit of $\mathbb{Z}(\langle y\rangle)$ for which $\varepsilon(z)=1$ and $\mu \in\{1,2,3,4\}$. But $z=u^{n} y^{m}$ for some $m, n$ and there exists $\nu \in\{0,1,2,3,4\}$ such that $\alpha^{\nu}(y)=y^{\mu}$ and hence if $\pi(x)=x z$ we have $\pi=\rho^{n} \sigma^{m} \alpha^{\nu}$ and if $\pi(x)=x^{-1} z$ we have $\pi=\tau \rho^{n} \sigma^{m} \alpha^{v}$. Hence, indeed, we have

$$
\text { Aut }+\mathbb{Z}(G)=\langle\alpha, \sigma, \tau, \rho\rangle
$$


We now consider relations in Aut $+\mathbb{Z}(G)$ additional to those of Aut $(G)$. We claim that $\rho^{-1} \alpha^{2} \rho \alpha^{2}=\sigma$. To see this we have

$$
\begin{aligned}
\left(\rho^{-1} \alpha^{2} \rho \alpha^{2}\right)(x) & =\rho^{-1} \alpha^{2} \rho(x)=\rho^{-1} \alpha^{2}(x u)=\rho^{-1}(x y u) \\
& =x u^{-1} y u=x y \\
\left(\rho^{-1} \alpha^{2} \rho \alpha^{2}\right)(y) & =\rho^{-1} \alpha^{2} \rho\left(y^{4}\right)=\rho^{-1} \alpha^{2}\left(y^{4}\right)=\rho^{-1}\left(y^{16}\right) \\
& =\rho^{-1}(y)=y
\end{aligned}
$$

giving the desired result. Let $H=\langle\alpha, \rho, \sigma\rangle=\langle\alpha, \rho\rangle$. We may verify that $\rho$ centralizes $\sigma$ and so $\langle\sigma\rangle$ is a normal subgroup of $H$ of order 5 . Further, again by direct calculation, we have

$$
\alpha^{-1} \rho \alpha=\rho^{-1} \sigma^{3}
$$

Thus $H /\langle\sigma\rangle$ is a non-trivial extension of an infinite cyclic group by an element of order 4. Collecting together our information on $\mathrm{Aut}_{+} \mathbb{Z}(G)$ we have finally

$$
\text { Aut }+\mathbb{Z}(G)=\langle\alpha, \rho, \tau, \sigma\rangle
$$

where

$$
\begin{gathered}
\tau^{2}=\imath, \quad \tau^{-1} \alpha \tau=\alpha, \quad \tau^{-1} \sigma \tau=\sigma^{4} \\
\alpha^{4}=\imath, \quad \sigma=\rho^{-1} \alpha^{2} \rho \alpha^{2}, \quad \sigma^{5}=\imath, \\
\alpha^{-1} \sigma \alpha=\sigma^{3}, \quad \rho^{-1} \sigma \rho=\sigma \\
\alpha^{-1} \rho \alpha=\rho^{-1} \sigma^{3} .
\end{gathered}
$$

We remark that Aut $+\mathbb{Z}(G)$ and $A u t \mathbb{Z}(G)$ are both polycyclic and so are both residually finite. In fact we may now prove, although we do not need the result, that $\operatorname{Aut} \mathbb{Z}(G)=$ Aut $+\mathbb{Z}(G) \times\langle\lambda\rangle$.

\section{The centre of $R(G)$}

The centre, $Z(R(G))$, of $R(G)$ is the $R$-linear subspace spanned by the class-sums of finite classes. Thus $Z(R(G))$ is trivial if and only if no element of $G$, other than the identity, has a finite number of conjugates (see [3], pp. 113-115). We denote the centre of Aut $R(G)$ by $Z(A u t R(G)$ ) and utilise essentially the proof of the result to which we wish to find the analogue. We reproduce the modified proof for the sake of completeness.

Theorem 4.1. Let $Z(R(G))$ be trivial. Then $Z($ Aut $R(G)$ ) is also trivial. 
Proof. Let $\theta \in Z($ Aut $R(G))$. Let $x \in G$ and let $\rho_{x}: g \rightarrow x^{-1} g x(g \in G)$ be the inner automorphism of $G$ induced by $x$. Then for all $a \in R(G)$ we have $\left(\theta \rho_{x}\right)\left(\theta^{-1}(a)\right)=\left(\rho_{x} \theta\right)\left(\theta^{-1}(a)\right)$ and thus $(\theta(x))^{-1} a \theta(x)=x^{-1} a x$ from which we conclude that $\theta(x) x^{-1} \in Z(R(G))$ and so $\theta(x)=x$. Since $x$ is an arbitrary element of $G$ we have $\theta(w)=w$ for all $w \in R(G)$ and so $\theta=t$.

\section{REFERENCES}

1. G. Baumslag, Automorphism groups of residually finite groups, J. London Math. Soc. 38 (1963), 117-118.

2. A. G. Kurosh, Theory of Groups, Vol. I (Chelsea Publishing, New York, 1955).

3. D. S. PASSMAN, The Algebraic Structure of Group Rings (Wiley-Interscience, New York, 1977).

4. S. K. Sehgal, Topics in Group Rings (Marcel Dekker, New York, 1978).

Department of Mathematics

University of StrathClyde

Glasgow G1 1XH

SCOTLAND 